\title{
EFEITO DE DOSES DE NITROGÊNIO, FÓSFORO E POTÁSSIO EM DUAS CULTIVARES DE CUPUAÇU, COM E SEM SEMENTES, NA REGIÃO DA AMAZÔNIA CENTRAL ${ }^{1}$
}

\author{
SONIA SENA ALFAIA ${ }^{2} \&$ MARTA IRIA DA COSTA AYRES ${ }^{3}$
}

\begin{abstract}
RESUMO - Com o objetivo de estudar o efeito de doses de N, P e K na produção e na nutrição mineral de duas cultivares de cupuaçuzeiro (Theobroma grandiflorum), um experimento foi instalado em Argissolo Vermelho-Amarelo na região da Amazônia Central. O delineamento experimental utilizado foi o de blocos casualizados, em parcelas subdivididas, com três repetições. Os tratamentos estudados nas parcelas foram: $\mathrm{N}_{1} \mathrm{P}_{1} \mathrm{~K}_{1} ; \mathrm{N}_{1} \mathrm{P}_{1} \mathrm{~K}_{0} ; \mathrm{N}_{1} \mathrm{P}_{0} \mathrm{~K}_{1} ; \mathrm{N}_{0}$ $\mathrm{P}_{1} \mathrm{~K}_{1} ; \mathrm{N}_{1} \mathrm{P}_{1} \mathrm{~K}_{2} ; \mathrm{N}_{1} \mathrm{P}_{2} \mathrm{~K}_{1}$ e $\mathrm{N}_{2} \mathrm{P}_{1} \mathrm{~K}_{1}$; e, nas subparcelas, as duas cultivares de cupuaçu: com e sem sementes. As doses de 0; 1 e 2 de N, $\mathrm{P}$ e $\mathrm{K}$ foram, respectivamente, de $0 ; 60$ e $120 \mathrm{~kg} \mathrm{ha}^{-1} \mathrm{de} \mathrm{N}$, na forma de uréia; $0 ; 100$ e $200 \mathrm{~kg} \mathrm{ha}^{-1} \mathrm{de}_{2} \mathrm{O}_{5}$, na forma de superfosfato triplo, e $0 ; 80$ e $160 \mathrm{~kg} \mathrm{ha}{ }^{-1}$ de $\mathrm{K}_{2} \mathrm{O}$, na forma de cloreto de potássio. O presente trabalho não possibilitou uma recomendação oficial de adubação para o cupuaçu, mas apresentou tendências das necessidades nutricionais da cultura. Considerando a produção média das três safras analisadas, não houve efeito da adubação nitrogenada na produção de frutos nas duas cultivares, assim como não houve da adubação com a dose mais elevada de fósforo. Contudo, os efeitos da aplicação de potássio apresentaram-se mais lineares e positivos, principalmente para a cultivar com sementes. Os menores valores de produção de frutos foram obtidos nos tratamentos com ausência de fósforo.
\end{abstract}

Termos para indexação: Theobroma grandiflorum, adubação, nutrição mineral, absorção nutrientes.

\section{EFFECTS OF NITROGEN, PHOSPHORUS AND POTASSIUM LEVELS ON OF TWO CUPUAÇU CULTIVARS, SEEDED AND SEED-LESS IN CENTRAL AMAZONIA}

\begin{abstract}
The effects of N, P and K levels on yield and mineral nutrition of two cupuaçu (Theobroma grandiflorum) cultivars were studied in an experiment on a Ultissol in Central Amazonia. A randomized block design with split-plots and three replications was used. The treatments applied on the plots were: $\mathrm{N}_{1} \mathrm{P}_{1} \mathrm{~K}_{1} ; \mathrm{N}_{1} \mathrm{P}_{1} \mathrm{~K}_{0} ; \mathrm{N}_{1} \mathrm{P}_{0} \mathrm{~K}_{1} ; \mathrm{N}_{0} \mathrm{P}_{1} \mathrm{~K}_{1} ; \mathrm{N}_{1} \mathrm{P}_{1} \mathrm{~K}_{2} ; \mathrm{N}_{1} \mathrm{P}_{2} \mathrm{~K}_{1}$ e $\mathrm{N}_{2} \mathrm{P}_{1} \mathrm{~K}_{1}$; and on the split-plots: two cupuaçu cultivars, seeded and seed-less. The 0,1 and 2 levels of N, $\mathrm{P}$ and $\mathrm{K}$ were, respectively, 0,60 and $120 \mathrm{~kg} \mathrm{ha}^{-1}$ of $\mathrm{N}$ as urea, 0,100 and $200 \mathrm{~kg} \mathrm{ha}^{-1}$ of $\mathrm{P}_{2} \mathrm{O}_{5}$ as treble superfosfate, and 0,80 and $160 \mathrm{~kg} \mathrm{ha}^{-1}$ of $\mathrm{K}_{2} \mathrm{O}$, as $\mathrm{KCl}$. Based on this study, it was not possible to make formal recommendations for fertilization of cupuaçu, however, trends in mineral nutrient requirements for this crop were evident. Based on the 3year mean yield, nitrogen fertilizer had no effect on crop yields, neither $\mathrm{P}$ fertilizer at high levels. However, a positive linear yield response to K application was observed, principally for the seeded cultivar. The lowest yield occurred in the absence of $P$.
\end{abstract}

Index term: Theobroma grandiflorum, fertilization, mineral nutrition, available nutrients.

\section{INTRODUÇÃO}

O cultivo do cupuaçu tem se tornado uma prática de grande importância econômica e social na Amazônia. No entanto, o reduzido número de pesquisa sobre o comportamento agronômico dessa espécie tem limitado a expansão da área cultivada na região. É uma cultura que apresenta grande potencial, porém a produtividade precisa ser elevada em relação aos níveis atuais, que é extremamente variável. Em plantações bem manejadas, a produtividade média situa-se em torno de 15 frutos/ planta/ano (Carvalho et al., 1999). A maioria dos solos, onde se têm estabelecido os plantios de cupuaçu na região, é de baixa fertilidade natural, e a adubação, quando realizada, é feita de forma empírica, sendo poucos os estudos feitos neste sentido (Cravo \& Souza, 1996).

A nutrição adequada das plantas de cupuaçu é fundamental para manter um crescimento vigoroso e elevadas produtividades, uma vez que colheitas sucessivas, sem a reposição dos nutrientes, poderão causar esgotamento do solo, tornando-se prejudiciais à cultura. Embora existam limitações devido à influência de outros fatores ainda não resolvidos e que influenciam na produção, tais como, genético, variação sazonal, ataques de pragas e doenças, os agricultores que se dedicam ao cultivo do cupuaçu, reconhecem a importância da adubação para o aumento da produtividade, mas enfrentam o problema da inexistência de pesquisas que possibilitem a indicação de fórmulas e doses agronomicamente corretas e economicamente viáveis. Atualmente, grande parte das informações utilizadas para o cultivo do cupuaçu é baseada em trabalhos realizados com o cacaueiro (Theobroma cacao), devido à proximidade taxonômica destas culturas. Alguns estudos têm demonstrado que o potássio é um dos nutrientes mais absorvidos pelas plantas de cupuaçu e também o que se exporta em maior quantidade com os frutos (Silva \& Silva, 1986; Venturieri, 1993; Cravo \& Souza, 1996). Para cada tonelada de frutos colhida, são exportados $3,7 \mathrm{~kg}$ de N; $0,24 \mathrm{~kg}$ de P, e 5,6 kg de K (Silva \& Silva, 1986). O K também é o nutriente que se encontra em maior quantidade no tecido do cacau, sendo que a maior parte fica retida na casca do fruto (Rodrigues, 1983).

Considerando o reduzido número de trabalhos com adubação de cupuaçu na literatura nacional e tomando-se como base os resultados das pesquisas com adubação do cacaueiro, o presente trabalho teve como objetivo estudar o efeito de nitrogênio, fósforo e potássio, na cultura do cupuaçu, assim como comparar a produção de duas cultivares de cupuaçuzeiros submetidas a diferentes níveis de adubação.

\section{MATERIAL E MÉTODOS}

O experimento foi instalado na Estação Experimental de Fruticultura Tropical do Instituto Nacional de Pesquisas da Amazônia INPA, em solo classificado como Argissolo Vermelho-Amarelo, textura média (Ranzanif́, 1980), em um plantio de cupuaçu com 10 anos de idade. As principais características químicas do solo encontram-se na Tabela 1. O clima da região é quente úmido, com duas estações bem distintas: uma chuvosa, de novembro a maio e outra menos chuvosa, de junho a outubro. O delineamento experimental utilizado foi o de blocos casualizados, em parcelas subdivididas, com três repetições. Os tratamentos estudados nas parcelas foram: $\mathrm{N}_{1} \mathrm{P}_{1} \mathrm{~K}_{1} ; \mathrm{N}_{1} \mathrm{P}_{1} \mathrm{~K}_{0} ; \mathrm{N}_{1} \mathrm{P}_{0} \mathrm{~K}_{1}$; $\mathrm{N}_{0} \mathrm{P}_{1} \mathrm{~K}_{1} ; \mathrm{N}_{1} \mathrm{P}_{1} \mathrm{~K}_{2} ; \mathrm{N}_{1} \mathrm{P}_{2} \mathrm{~K}_{1}$ e $\mathrm{N}_{2} \mathrm{P}_{1} \mathrm{~K}_{1} \mathrm{e}$, nas subparcelas as duas cultivares de cupuaçu: o cupuaçu "redondo", o mais comum na região, e a do tipo "mamau", cujos frutos não apresentam sementes (Calzavara et al., 1984). As doses de 0; 1 e 2 de N, P e K foram, respectivamente, de 0; 60 e $120 \mathrm{~kg}$ ha ${ }^{-1} \mathrm{de} \mathrm{N}$, na forma de uréia; $0 ; 100$ e $200 \mathrm{~kg} \mathrm{ha}^{-1} \mathrm{de}_{2} \mathrm{O}_{5}$, na forma de

\footnotetext{
${ }^{1}$ (Trabalho 182/2003). Recebido: 04/12/2003. Aceito para publicação: 02/08/2004. Trabalho financiado com recursos do Programa Piloto para Proteção das Florestas Tropicais, PPD/PPG-7.

${ }^{2}$ Eng. Agrôn., Dra., Pesquisadora do Instituto Nacional de Pesquisas da Amazônia-INPA, Av. André Araújo, 2936-Petropólis, Caixa Postal 478, 69.011-970, ManausAM, fone (0xx92)6431853, e-mail: sonia@inpa.gov.br.

${ }^{3}$ Eng. Agrôn., D.Sc., Bolsista de DTI/PCI, do Instituto Nacional de Pesquisas da Amazônia, e-mail: iria@inpa.gov.br.
} 
superfosfato triplo, e 0 ; 80 e $160 \mathrm{~kg} \mathrm{ha}^{-1}$ de $\mathrm{K}_{2} \mathrm{O}$, na forma de cloreto de potássio. Três meses antes da aplicação dos fertilizantes, em setembro de 1997, aplicou-se em todas as parcelas o equivalente a $2 \mathrm{t} \mathrm{ha}^{-1} \mathrm{de}$ calcário dolomítico para a correção da acidez do solo e fornecimento de cálcio e magnésio, tomando-se como base a recomendação para outros cultivos na região (Cravo e Smyth, 1991). Todas as parcelas receberam ainda uma adubação complementar com micronutrientes na forma de fritas (FTE), na quantidade de $50 \mathrm{~kg}$ da mistura por hectare (120g/planta), aplicados no mesmo período da adubação com NPK, realizada em dezembro de 1997. No segundo e terceiro anos, em setembro de 1998 e 1999, foi efetuada apenas a adubação com NPK, nas mesmas quantidades da primeira adubação.

TABELA 1 - Principais características químicas do Argissolo VermelhoAmarelo da área experimental, localizada na região de Manaus-AM, 1987.

\begin{tabular}{cccccccccc}
\hline $\begin{array}{c}\text { Profundidade } \\
(\mathrm{cm})\end{array}$ & $\mathrm{pH} \mathrm{em}$ & $\mathrm{Ca}$ & $\mathrm{Mg}$ & $\mathrm{Al}$ & $\mathrm{K}$ & $\mathrm{P}$ & $\begin{array}{c}\mathrm{N} \\
\text { total }\end{array}$ & $\mathrm{M} . \mathrm{O}$ \\
\hline & $\mathrm{H}_{2} \mathrm{O}$ & & & \multicolumn{6}{c}{} \\
$0-20$ & 4,3 & 0,07 & 0,07 & 0,83 & 0,06 & 3,69 & 0,93 & 26 \\
$20-40$ & 4,2 & 0,05 & 0,04 & 0,90 & 0,05 & 1,89 & 0,69 & 19 \\
\hline
\end{tabular}

De cada cultivar, foram utilizadas cinco plantas úteis alinhadas por parcela, com o espaçamento de cinco metros entre plantas e seis metros entre as linhas. No final de cada safra de cupuaçu, foram coletadas amostras de solo e de folhas para análise química dos principais nutrientes que foram determinados segundo a metodologia descrita pela EMBRAPA (1997). Para análise química foliar, foram coletadas amostras de $3^{\mathrm{a}}$ folha, tomadas do ápice do ramo da altura média, seguindo-se a mesma recomendação utilizada para o cacau (Malavolta, 1989), num total de cinco folhas de cada planta útil das parcelas. O experimento recebeu todos os tratos culturais necessários e foi avaliado por três anos consecutivos.

A cada safra, foram tomados os dados de produção relacionados com o número e peso de frutos das cinco plantas úteis de cada parcela. Os resultados obtidos foram avaliados pela análise de variância, aplicando-se análise de regressão para as doses. As comparações entre as médias dos parâmetros avaliados foram realizadas pelo teste de Tukey, ae nível de 5\% de probabilidade.

\section{RESULTADOS E DISCUSSÃO}

A Tabela 2 apresenta os resultados das três safras de cupuaçu avaliadas. Ressalta-se que o prolongado período de seca devido ao fenômeno do "El Niño", em meados do ano de 1997, induziu a queda de grandes quantidades de flores e frutos novos, causando severos danos na safra de 1997-1998. Por outro lado, a safra de 1999-2000 também foi seriamente afetada pela vassoura-de-bruxa. Assim, dentre as três safras analisadas, a do ano de 1998-1999 foi a que mais expressou o efeito dos nutrientes aplicados na produção de frutos, sem haver interferência de outros fatores externos. Segundo Carvalho et al. (1999), aproximadamente $75 \%$ dos frutos de cupuaçu em formação podem ser abortados como conseqüência de problemas nutricionais, disponibilidade hídrica e ataque de pragas e doenças, entre outros fatores.

Os resultados mostraram que, nas três safras avaliadas, (19981999, 1997-1998 e 1999-2000) a produção de frutos foi significativamente mais elevada nas plantas de cupuaçu com sementes. No entanto, na safra de 1998-1999, apesar da maior produção em número de frutos na cultivar com sementes, o peso dos frutos em $\mathrm{kg} / \mathrm{planta}$ foi

TABELA 2 - Efeito de doses de N, Pe K na produção de três safras de duas cultivares de cupuaçu, em um Argissolo Vermelho-Amarelo da Amazônia Central.

\begin{tabular}{|c|c|c|c|c|c|c|}
\hline \multirow{2}{*}{ Tratamentos } & \multicolumn{3}{|c|}{ Cupuaçu sem sementes } & \multicolumn{3}{|c|}{ Cupuaçu com sementes } \\
\hline & $\mathrm{N}^{\mathrm{o}}$ de frutos & $\begin{array}{l}\text { Peso de frutos } \\
\text { (kg/planta) }\end{array}$ & $\begin{array}{l}\text { Peso médio de } \\
\text { frutos }(\mathrm{g})\end{array}$ & $\mathrm{N}^{0}$ de frutos & $\begin{array}{l}\text { Peso de frutos } \\
(\mathrm{kg} / \mathrm{planta})\end{array}$ & $\begin{array}{l}\text { Peso médio de } \\
\text { frutos }(\mathrm{g})\end{array}$ \\
\hline & \multicolumn{6}{|c|}{ Safra de 1997/1998 } \\
\hline $\mathrm{N}_{1} \mathrm{P}_{1} \mathrm{~K}_{2}$ & 4,0 & 4,27 & 1068 & 13,7 & 10,91 & 795 \\
\hline $\mathrm{N}_{1} \mathrm{P}_{1} \mathrm{~K}_{1}$ & 3,0 & 3,81 & 1269 & 12,0 & 8,04 & 668 \\
\hline $\mathrm{N}_{2} \mathrm{P}_{1} \mathrm{~K}_{1}$ & 1,0 & 1,24 & 1235 & 9,3 & 9,02 & 966 \\
\hline $\mathrm{N}_{1} \mathrm{P}_{2} \mathrm{~K}_{1}$ & 1,0 & 0,88 & 880 & 9,1 & 6,79 & 743 \\
\hline $\mathrm{N}_{0} \mathrm{P}_{1} \mathrm{~K}_{1}$ & 1,3 & 1,89 & 1418 & 14,8 & 11,24 & 761 \\
\hline $\mathrm{N}_{1} \mathrm{P}_{1} \mathrm{~K}_{0}$ & 5,0 & 3,23 & 646 & 13,0 & 9,21 & 710 \\
\hline $\mathrm{N}_{1} \mathrm{P}_{0} \mathrm{~K}_{1}$ & 0,0 & 0,00 & 0 & 10,0 & 6,91 & 690 \\
\hline \multirow[t]{2}{*}{ Média } & $2,1 \mathrm{~B}^{(2)}$ & $2,14 \mathrm{~B}^{(1)}$ & 936 & $12,3 \mathrm{~A}^{(2)}$ & $8,73 \mathrm{~A}^{(1)}$ & 736 \\
\hline & \multicolumn{6}{|c|}{ Safra de 1998/1999 } \\
\hline $\mathrm{N}_{1} \mathrm{P}_{1} \mathrm{~K}_{2}$ & 14,7 & $24,59 \mathrm{a}$ & 1674 & $21,5 \mathrm{a}$ & $21,14 \mathrm{a}$ & 983 \\
\hline $\mathrm{N}_{1} \mathrm{P}_{1} \mathrm{~K}_{1}$ & 14,4 & $24,01 \mathrm{a}$ & 1667 & $21,1 \mathrm{a}$ & $18,55 \mathrm{ab}$ & 878 \\
\hline $\mathrm{N}_{2} \mathrm{P}_{1} \mathrm{~K}_{1}$ & 11,0 & $18,54 \mathrm{ab}$ & 1686 & $21,5 \mathrm{a}$ & $18,22 \mathrm{abc}$ & 847 \\
\hline $\mathrm{N}_{1} \mathrm{P}_{2} \mathrm{~K}_{1}$ & 12,8 & $20,89 \mathrm{ab}$ & 1631 & $18,3 \mathrm{ab}$ & $16,65 \mathrm{abc}$ & 908 \\
\hline $\mathrm{N}_{0} \mathrm{P}_{1} \mathrm{~K}_{1}$ & 14,3 & $22,70 \mathrm{ab}$ & 1592 & $19,4 \mathrm{ab}$ & $15,90 \mathrm{abc}$ & 820 \\
\hline $\mathrm{N}_{1} \mathrm{P}_{1} \mathrm{~K}_{0}$ & 10,0 & $15,22 \mathrm{ab}$ & 1522 & $15,3 \mathrm{ab}$ & $13,07 \mathrm{bc}$ & 854 \\
\hline $\mathrm{N}_{1} \mathrm{P}_{0} \mathrm{~K}_{1}$ & 7,5 & $10,52 \mathrm{~b}$ & 1402 & $10,6 \mathrm{~b}$ & $10,64 \mathrm{c}$ & 1001 \\
\hline \multirow[t]{2}{*}{ Média } & $12,3 \mathrm{~B}^{(2)}$ & $19,50 \mathrm{~A}^{(1)}$ & 1588 & $18,3 \mathrm{~A}^{(2)}$ & $16,30 \mathrm{~B}^{(1)}$ & 835 \\
\hline & \multicolumn{6}{|c|}{ Safra de 1999/2000 } \\
\hline $\mathrm{N}_{1} \mathrm{P}_{1} \mathrm{~K}_{2}$ & 3,3 & 4,38 & 1372 & $12,3 \mathrm{ab}$ & $13,48 \mathrm{a}$ & 1099 \\
\hline $\mathrm{N}_{1} \mathrm{P}_{1} \mathrm{~K}_{1}$ & 3,7 & 5,19 & 1610 & $11,0 \mathrm{abc}$ & $10,14 \mathrm{ab}$ & 922 \\
\hline $\mathrm{N}_{2} \mathrm{P}_{1} \mathrm{~K}_{1}$ & 5,0 & 4,23 & 1361 & $5,8 \quad \mathrm{c}$ & $4,34 \mathrm{c}$ & 743 \\
\hline $\mathrm{N}_{1} \mathrm{P}_{2} \mathrm{~K}_{1}$ & 3,3 & 4,87 & 1461 & $13,7 \mathrm{a}$ & $13,46 \mathrm{a}$ & 981 \\
\hline $\mathrm{N}_{0} \mathrm{P}_{1} \mathrm{~K}_{1}$ & 2,7 & 3,81 & 1429 & $10,9 \mathrm{abc}$ & $9,48 \mathrm{abc}$ & 867 \\
\hline $\mathrm{N}_{1} \mathrm{P}_{0} \mathrm{~K}_{1}$ & 1,7 & 2,39 & 1432 & 7,3 bc & $7,64 \mathrm{bc}$ & 1091 \\
\hline $\mathrm{N}_{1} \mathrm{P}_{1} \mathrm{~K}_{0}$ & 3,0 & 3,87 & 1290 & $6,4 \quad c$ & 5,39 bc & 841 \\
\hline Média & $3,2 \mathrm{~B}^{(2)}$ & $4,10 \mathrm{~B}^{(1)}$ & 1456 & $9,0 \mathrm{~A}^{(2)}$ & $9,48 \mathrm{~A}^{(1)}$ & 937 \\
\hline
\end{tabular}

Médias seguidas por letras minúsculas distintas nas colunas e letras maiúsculas distintas nas linhas (para uma mesma característica) diferem entre si, ao nível de 5\% de probabilidade, pelo teste de Tukey.

${ }^{(1)}$ Teste de média para a característica kg de frutos/planta

${ }^{(2)}$ Teste de média para a característica $\mathrm{n}^{\mathrm{o}}$ de frutos 


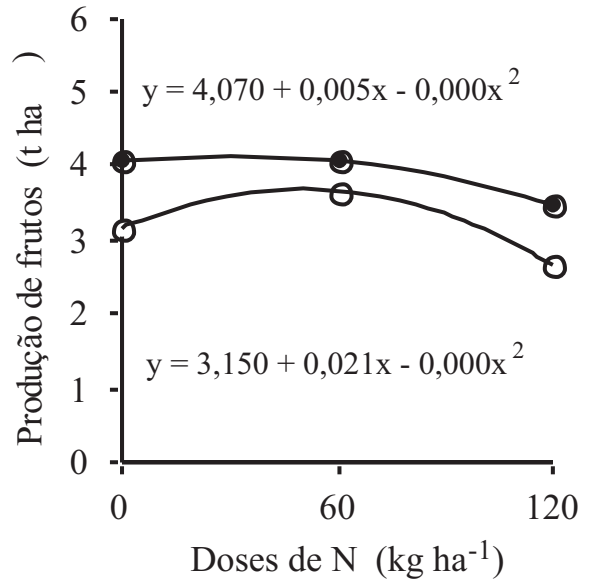

0

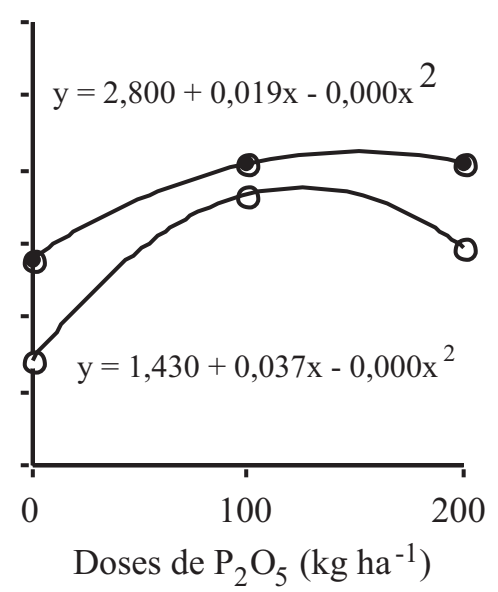

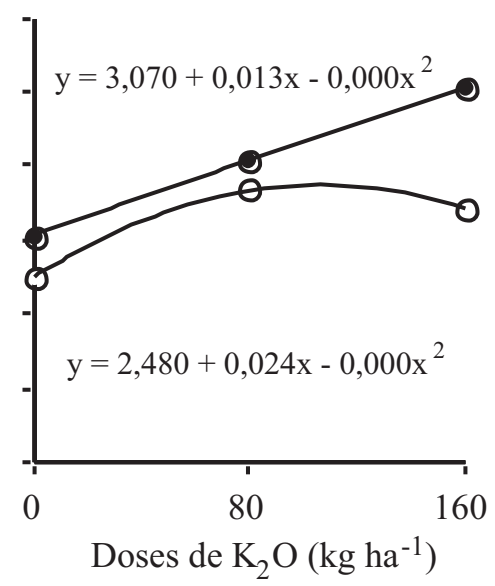

FIGURA 1- Relação entre a produção média de três safras de duas cultivares de cupuaçu e as quantidades de N, Pe K aplicadas em um Argissolo Vermelho-Amarelo da Amazônia Central.

significativamente mais elevado na cultivar sem sementes. Em todas as safras avaliadas, os frutos das plantas sem sementes apresentaram um peso médio que foi quase o dobro do peso dos frutos das plantas com sementes.

Na safra de 1997-1998, a ausência de resposta aos tratamentos pode ter sido mais uma influência do "El Niño". Na safra de 1998-1999, para as duas cultivares, as maiores produções foram obtidas na presença da dose 2 de $\mathrm{K}$ e também no tratamento com a dose 1 de N, P e K. Por outro lado, as menores produções foram obtidas nos tratamentos com ausência de $\mathrm{P}$, com diferenças significativas em relação aos tratamentos onde se obtiveram as maiores produções.

Na safra de 1999-2000, a cultivar sem sementes mostrou maior suscetibilidade ao ataque da vassoura-de-bruxa, apresentando acentuada redução na produção de frutos em relação à safra anterior. Para essa cultivar, não houve efeito dos nutrientes aplicados. Quanto à cultivar com sementes, as produções mais elevadas foram obtidas na presença das doses mais elevadas de $\mathrm{P}$ e $\mathrm{K}$ e produções mais baixas nos tratamentos com ausência de $\mathrm{P}$ e K, além do tratamento com alta dose de $\mathrm{N}$.

A Figura 1 apresenta o efeito das doses de $0 ; 1$ e 2 de $N, \mathrm{P}_{2} \mathrm{O}_{5}$ e $\mathrm{K}_{2} \mathrm{O}$ sobre a produção de frutos, considerando a média das três safras analisadas. Os resultados indicam que, para as duas cultivares, não há necessidade de adubação nitrogenada, assim como de adubação com doses elevadas de fósforo. Contudo, os efeitos para a aplicação de potássio apresentaram-se mais lineares e positivos, principalmente para a cultivar de cupuaçu com sementes.

Ausência de resposta à adubação nitrogenada na produção de frutos de cupuaçu também foi observada por Schroth et al. (2001), em um consórcio agroflorestal na região da Amazônia Central. Segundo os autores, a ausência de resposta poderia estar relacionada com a alta taxa de mineralização de $\mathrm{N}$ no solo devido à utilização da Puerária phaseoloides, como leguminosa de cobertura no plantio. Nas condições do presente experimento, é provável que o uso de plantas de cobertura, incluindo a presença de uma leguminosa, como o Desmodium ovalifolium, possa também ter contribuído para o fornecimento de $\mathrm{N}$ para as plantas de cupuaçu. Por outro lado, o efeito não-significativo da adubação nitrogenada pode também estar relacionado com a alta taxa de lixiviação de N nesses solos, conforme relatado por Alfaia (2000), a qual é favorecida, principalmente, pelo baixo teor de argila do solo, onde foi conduzido o ensaio.

A Figura 1 mostra uma tendência quadrática de resposta da produção à aplicação de doses crescentes de $\mathrm{P}$. O mesmo tipo de resposta tem sido relatado para o cacaueiro, em função da aplicação de doses crescentes de $\mathrm{P}$, havendo uma sincronia entre os dados de diâmetro do caule, produção de amêndoas secas, nível de P no solo e na planta. Para essa espécie, a melhor dosagem de $\mathrm{P}_{2} \mathrm{O}_{5}$ nos solos da Amazônia, segundo Morais \& Pereira (1986), situou-se em torno de $120 \mathrm{~kg} \mathrm{ha}^{-1}$. Embora não tenha sido observada resposta à aplicação da dose mais elevada de $\mathrm{P}$, esse parece ser o principal nutriente que pode limitar a produção, pois os menores valores de produção de frutos de cupuaçu foram obtidos no tratamento com ausência de $\mathrm{P}$, principalmente a produção da cultivar sem sementes.

Com relação ao efeito do $\mathrm{K}$, os resultados encontrados confirmam a importância desse nutriente para a cultura do cupuaçu, conforme tem sido observado por outros autores na região (Silva \& Silva, 1986; Venturieri, 1993; Cravo \& Souza, 1996). O K é um dos elementos mais exportados nas colheitas, principalmente através das cascas. Cravo \& Souza (1996) relatam que a exportação desse macronutriente com os frutos gira em torno de $4.960 \mathrm{~g} \mathrm{t}^{-1}$, enquanto Silva \& Silva (1986) encontraram $5.590 \mathrm{~g} \mathrm{t}^{-1}$. Mais recentemente, Santos (2003) mostrou o efeito favorável da utilização do resíduo da casca de cupuaçu como fonte de K para as plantas de cupuaçu em consórcios agroflorestais do Projeto RECA em Rondônia.

Os valores de N, P, K, Ca e Mg determinados pela análise foliar nos diversos tratamentos encontram-se na Tabela 3. Os resultados mostram que, durante todos os anos, houve um incremento significativo da concentração de $\mathrm{P}$ nas folhas da planta com o aumento da dose desse nutriente. Existem poucos trabalhos na literatura com interpretação de resultados analíticos em plantas de cupuaçu na região. Nesse trabalho, com exceção do tratamento com ausência de $\mathrm{P}$, todos os demais apresentaram concentração de $\mathrm{P}$ na folha dentro da faixa observada por Schroth et al. (2001) e por Figueredo et al. (2000) em amostras de folhas novas ( $3^{\text {a }}$ folha) de cupuaçuzeiros, coletadas no final da produção, na região da Amazônia Central. Os resultados mostram ainda que ocorreu um incremento significativo na absorção de $\mathrm{P}$ na presença da dose mais elevada de $\mathrm{N}$, indicando que este nutriente favorece a absorção de $\mathrm{P}$ pelo cupuaçuzeiro. O efeito benéfico da interação NP também tem sido encontrado para a cultura do cacau (Wessel, 1970; Morais et al., 1978; Morais \& Pereira, 1986).

No ano de 1999, também foi observado incremento significativo de $\mathrm{K}$ nas folhas em função do aumento da dose de $\mathrm{K}_{2} \mathrm{O}$ aplicada. Por outro lado, na maioria dos tratamentos, as concentrações de $\mathrm{K}$ nas folhas das plantas sem sementes foram significativamente mais elevadas do que nas folhas das plantas com sementes. Esses resultados sugerem que há maior exigência da cultivar sem sementes para esses nutrientes. Na maioria dos tratamentos, a concentração de K apresentou-se acima dos valores observados por Schroth et al. (2001) e por Figueredo et al. (2000). Os resultados da análise efetuada em 1999 mostram que ocorreu decréscimo acentuado na absorção de K, na presença da dose mais elevada de N. Segundo Morais \& Pereira (1986), o efeito depressivo da interação NK observado na cultura do cacau, na Amazônia, sugere a lixiviação intensa de K, que arrasta ânions do sistema solo, principalmente nitrato, ocasionando desbalanço nutricional na planta. 
TABELA 3 - Concentração de N, P, K, Ca e Mg nas folhas de cupuaçuzeiros, em um Argissolo Vermelho-Amarelo da Amazônia Central.

\begin{tabular}{|c|c|c|c|c|c|c|c|c|c|c|}
\hline \multirow[b]{2}{*}{ Doses } & \multicolumn{2}{|c|}{$\mathrm{N}$} & \multicolumn{2}{|c|}{$\mathrm{P}$} & \multicolumn{2}{|c|}{$\mathrm{K}$} & \multicolumn{2}{|c|}{$\mathrm{Ca}$} & \multicolumn{2}{|c|}{$\mathrm{Mg}$} \\
\hline & $\begin{array}{c}\text { Com } \\
\text { semente }\end{array}$ & $\begin{array}{c}\text { Sem } \\
\text { semente }\end{array}$ & $\begin{array}{c}\text { Com } \\
\text { semente }\end{array}$ & $\begin{array}{c}\text { Sem } \\
\text { semente }\end{array}$ & $\begin{array}{c}\text { Com } \\
\text { semente }\end{array}$ & $\begin{array}{c}\text { Sem } \\
\text { semente }\end{array}$ & $\begin{array}{c}\text { Com } \\
\text { semente }\end{array}$ & $\begin{array}{c}\text { Sem } \\
\text { semente }\end{array}$ & $\begin{array}{c}\text { Com } \\
\text { semente }\end{array}$ & $\begin{array}{c}\text { Sem } \\
\text { semente }\end{array}$ \\
\hline & & & & & 1998 & $\mathrm{~kg}^{-1}---\cdot---\cdot$ & & 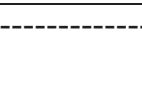 & & \\
\hline $\mathrm{N}_{0}$ & 15,8 & 15,4 & 1,08 & 1,07 & 6,97 & 9,72 & 3,71 & 3,03 & 1,75 & 1,97 \\
\hline $\mathrm{N}_{1}$ & 16,6 & 17,2 & 1,09 & 1,15 & 6,97 & 9,17 & 3,07 & 2,51 & 1,83 & 1,70 \\
\hline $\mathrm{N}_{2}$ & 18,5 & 17,9 & 1,23 & 1,14 & 7,06 & 9,63 & 3,04 & 2,55 & 1,72 & 1,75 \\
\hline Média & 16,5 & 16,2 & 1,13 & 1,12 & $7,00 \mathrm{~B}$ & $9,50 \mathrm{~A}$ & $3,27 \mathrm{~A}$ & $2,69 \mathrm{~B}$ & 1,77 & 1,81 \\
\hline $\mathrm{P}_{0}$ & 16,1 & 15,5 & $0,93 \mathrm{~b}$ & $0,85 \mathrm{~b}$ & 7,88 & 11,00 & 3,10 & 2,70 & 1,85 & 1,78 \\
\hline $\mathrm{P}_{1}$ & 16,6 & 17,2 & $1,09 \mathrm{a}$ & $1,15 \mathrm{a}$ & 6,97 & 9,17 & 3,07 & 2,51 & 1,83 & 1,70 \\
\hline $\mathrm{P}_{2}$ & 18,0 & 16,7 & $1,19 \mathrm{a}$ & $1,05 \mathrm{a}$ & 6,23 & 8,98 & 4,50 & 3,49 & 1,89 & 1,79 \\
\hline Média & 16,9 & 16,5 & 1,07 & 1,02 & $7,02 \mathrm{~B}$ & $9,72 \mathrm{~A}$ & $3,56 \mathrm{~A}$ & $2,89 \mathrm{~B}$ & 1,86 & 1,75 \\
\hline $\mathrm{K}_{0}$ & 16,1 & 16,7 & 1,09 & 1,15 & 6,97 & 8,07 & 3,11 & 3,42 & 1,87 & 1,81 \\
\hline $\mathrm{K}_{1}$ & 16,6 & 17,2 & 1,09 & 1,15 & 6,97 & 9,17 & 3,07 & 2,51 & 1,83 & 1,70 \\
\hline $\mathrm{K}_{2}$ & 17,6 & 15,7 & 1,14 & 1,07 & 6,97 & 9,90 & 3,77 & 3,59 & 1,82 & 1,83 \\
\hline Média & 16,7 & 16,5 & 1,11 & 1,12 & $\begin{array}{l}6,97 \mathrm{~B} \\
1999\end{array}$ & $9,04 \mathrm{~A}$ & 3,32 & 3,17 & 1,84 & 1,78 \\
\hline $\mathrm{N}_{0}$ & 16,4 & 17,0 & $1,17 \mathrm{ab}$ & $1,11 \mathrm{~b}$ & 4,68 & 6,31 & 5,16 & 5,13 & 2,02 & 2,13 \\
\hline $\mathrm{N}_{1}$ & 16,6 & 16,9 & $1,05 \mathrm{~b}$ & $1,11 \mathrm{~b}$ & 4,68 & 5,16 & 4,60 & 6,73 & 1,76 & 2,08 \\
\hline $\mathrm{N}_{2}$ & 16,8 & 17,1 & $1,24 \mathrm{a}$ & $1,25 \mathrm{a}$ & 2,38 & 2,78 & 3,09 & 3,49 & 0,77 & 1,00 \\
\hline Média & 16,4 & 17,3 & 1,15 & 1,16 & $3,91 \mathrm{~B}$ & $4,75 \mathrm{~A}$ & 4,28 & 5,11 & 1,52 & 1,74 \\
\hline $\mathrm{P}_{0}$ & 16,0 & 17,6 & $0,94 \mathrm{c}$ & $0,91 \quad \mathrm{c}$ & 6,07 & 7,21 & 3,60 & 3,72 & 1,73 & 2,01 \\
\hline $\mathrm{P}_{1}$ & 16,6 & 16,9 & $1,05 \mathrm{~b}$ & $1,11 \mathrm{~b}$ & 4,68 & 5,16 & 4,60 & 6,73 & 1,76 & 2,08 \\
\hline $\mathrm{P}_{2}$ & 16,6 & 17,6 & $1,37 \mathrm{a}$ & $1,21 \mathrm{a}$ & 5,05 & 4,96 & 6,73 & 4,47 & 1,89 & 1,32 \\
\hline Média & 16,4 & 17,3 & 1,12 & 1,08 & 5,27 & 5,78 & 4,98 & 4,97 & 1,79 & 1,80 \\
\hline $\mathrm{K}_{0}$ & 16,1 & 16,8 & 1,27 & 1,27 & $4,36 \mathrm{~b}$ & $4,83 \mathrm{~b}$ & 4,90 & 6,65 & 2,10 & 2,24 \\
\hline $\mathrm{K}_{1}$ & 16,6 & 16,9 & 1,05 & 1,11 & $4,68 \mathrm{~b}$ & $5,16 \mathrm{~b}$ & 4,60 & 6,73 & 1,76 & 2,08 \\
\hline $\mathrm{K}_{2}$ & 16,9 & 17,6 & 1,25 & 1,20 & $5,16 \mathrm{a}$ & $6,20 \mathrm{a}$ & 2,82 & 3,49 & 1,92 & 2,22 \\
\hline Média & 16,5 & 17,1 & 1,19 & 1,19 & $\begin{array}{l}4,74 \\
2000\end{array}$ & 5,43 & 4,10 & 5,62 & 1,93 & 2,18 \\
\hline $\mathrm{N}_{0}$ & 16,5 & 16,9 & $1,10 \mathrm{~b}$ & $1,03 \mathrm{~b}$ & 5,28 & 8,33 & 4,98 & 4,65 & 2,02 & 1,89 \\
\hline $\mathrm{N}_{1}$ & 15,7 & 16,1 & $1,09 \mathrm{~b}$ & $0,99 \mathrm{~b}$ & 5,85 & 6,69 & 4,43 & 5,23 & 1,64 & 1,94 \\
\hline $\mathrm{N}_{2}$ & 16,4 & 16,5 & $1,30 \mathrm{a}$ & $1,13 \mathrm{a}$ & 7,02 & 7,84 & 4,37 & 5,01 & 1,68 & 1,82 \\
\hline Média & 16,9 & 16,6 & $1,16 \mathrm{~A}$ & $1,05 \mathrm{~B}$ & 6,05 & $7,61 \mathrm{~A}$ & 4,59 & 4,95 & 1,78 & 1,89 \\
\hline $\mathrm{P}_{0}$ & 16,1 & 16,1 & $0,80 \mathrm{c}$ & $0,75 \mathrm{c}$ & 6,45 & 7,22 & 3,53 & 4,14 & 1,49 & 1,77 \\
\hline $\mathrm{P}_{1}$ & 15,7 & 16,1 & $1,09 \mathrm{~b}$ & $0,99 \mathrm{~b}$ & 5,85 & 6,69 & 4,43 & 5,23 & 1,64 & 1,94 \\
\hline $\mathrm{P}_{2}$ & 17,1 & 16,6 & $1,26 \mathrm{a}$ & $1,14 \mathrm{a}$ & 5,57 & 6,65 & 5,37 & 4,85 & 1,84 & 1,97 \\
\hline Média & 16,3 & 16,5 & $1,05 \mathrm{~A}$ & $0,96 \mathrm{~B}$ & $5,95 \mathrm{~B}$ & $6,85 \mathrm{~A}$ & 4,44 & 4,67 & 1,66 & 1,90 \\
\hline $\mathrm{K}_{0}$ & 15,5 & 16,6 & 1,15 & 1,14 & 5,38 & 6,78 & 4,71 & 4,94 & 2,04 & 2,14 \\
\hline $\mathrm{K}_{1}$ & 15,7 & 16,1 & 1,09 & 0,99 & 5,85 & 6,69 & 4,43 & 5,23 & 1,64 & 1,94 \\
\hline $\mathrm{K}_{2}$ & 16,7 & 17,5 & 1,15 & 1,03 & 6,50 & 7,10 & 4,37 & 5,01 & 1,61 & 2,04 \\
\hline Média & $15,9 \mathrm{~B}$ & $16,7 \mathrm{~A}$ & $1,13 \mathrm{~A}$ & $1,05 \mathrm{~B}$ & $5,91 \mathrm{~B}$ & $6,86 \mathrm{~A}$ & 4,50 & 5,06 & 1,76 & 2,04 \\
\hline
\end{tabular}

Médias seguidas por letras minúsculas distintas nas colunas e letras maiúsculas distintas nas linhas (para um mesmo nutriente) diferem entre si, ao nível de 5\% de probabilidade, pelo teste de Tukey.

Com relação à concentração foliar de N, não houve diferença significativa entre os tratamentos. Esses resultados seguem a mesma tendência dos resultados obtidos por Schroth et al. (2001), que também observaram ausência de efeito da adubação nitrogenada na concentração de $\mathrm{N}$ nas folhas de cupuaçuzeiros.

As concentrações de $\mathrm{Ca}$ e $\mathrm{Mg}$ na maioria das amostras analisadas apresentaram-se abaixo dos teores encontrados por Figueredo et al. (2000) e Schroth et al. (2001), mostrando que esses nutrientes podem tornar-se limitantes nas próximas safras. Os resultados da análise do solo efetuada em 1988 (Tabela 4) mostram que a calagem aumentou o teor de $\mathrm{Ca}$ e $\mathrm{Mg}$ e reduziu a concentração do $\mathrm{Al}$ trocável no solo; no entanto, os resultados da análise química efetuada dois anos após mostra que houve um decréscimo acentuado no teor de $\mathrm{Ca}$ e $\mathrm{Mg}$, indicando a necessidade de reposição desses nutrientes no solo.

Os resultados da Tabela 5 mostram que as aplicações anuais de
$P$ fizeram com que esse nutriente se acumulasse significativamente no solo, ao contrário do $\mathrm{K}$, que apresentou, ao longo dos três anos, valores extremamente baixos, até mesmo abaixo do nível considerado crítico, segundo os critérios de Cochrane et al. (1984), o que pode estar relacionado com a exportação desse nutriente com a colheita dos frutos, assim como com a perda por lixiviação. Em estudos de ciclagem de nutrientes com a cultura do cacau, foi demonstrado que o Ké o nutriente que apresenta maior mobilidade, constituindo-se no principal fator limitante para o cultivo de cacau (Fassbender et al., 1985). Os resultados mostraram que ocorreu incremento no teor do $\mathrm{N}$ total do solo, provavelmente devido ao manejo da matéria orgânica pela incorporação anual ao solo dos resíduos das plantas de cobertura. Os resultados da Tabela 4 confirmam que, no final do experimento, ocorreu um incremento do teor de matéria orgânica do solo. 
TABELA 4 - Matéria orgânica, pH, Ca, Mg e Al no solo, em duas épocas de amostragem.

\begin{tabular}{|c|c|c|c|c|c|c|c|c|c|c|}
\hline \multirow{2}{*}{$\begin{array}{l}\text { Doses } \\
\text { NPK }\end{array}$} & \multicolumn{2}{|c|}{ M.O } & \multicolumn{2}{|c|}{$\mathrm{pH} \mathrm{em} \mathrm{H}_{2} \mathrm{O}$} & \multicolumn{2}{|c|}{$\mathrm{Ca}$} & \multicolumn{2}{|c|}{$\mathrm{Mg}$} & \multicolumn{2}{|c|}{$\mathrm{Al}$} \\
\hline & $0-20 \mathrm{~cm}$ & $20-40 \mathrm{~cm}$ & $0-20 \mathrm{~cm}$ & $20-40 \mathrm{~cm}$ & $0-20 \mathrm{~cm}$ & $20-40 \mathrm{~cm}$ & $0-20 \mathrm{~cm}$ & $20-40 \mathrm{~cm}$ & $0-20 \mathrm{~cm}$ & $20-40 \mathrm{~cm}$ \\
\hline & \multirow{2}{*}{\multicolumn{2}{|c|}{------ $\mathrm{g} \mathrm{kg}^{-1}$------- }} & & & \multirow{2}{*}{\multicolumn{5}{|c|}{--------------- $\mathrm{cmol}_{\mathrm{c}} \mathrm{kg}^{-1}$----------------- }} & \\
\hline & & & & & & & & & & \\
\hline $\mathrm{N}_{0}$ & 23,2 & 15,8 & 4,3 & 4,3 & 1,33 & 0,66 & 0,86 & 0,47 & 0,33 & 0,33 \\
\hline $\mathrm{N}_{1}$ & 24,9 & 16,6 & 4,3 & 4,2 & 2,90 & 0,93 & 1,25 & 0,52 & 0,33 & 0,33 \\
\hline $\mathrm{N}_{2}$ & 25,4 & 16,4 & 4,4 & 4,2 & 3,10 & 0,76 & 1,26 & 0,45 & 0,43 & 0,53 \\
\hline $\mathrm{P}_{0}$ & 26,2 & 16,8 & 4,2 & 4,4 & 2,56 & 0,94 & 1,23 & 0,57 & 0,33 & 0,27 \\
\hline $\mathrm{P}_{1}$ & 24,4 & 15,9 & 4,2 & 4,3 & 2,64 & 0,88 & 1,16 & 0,52 & 0,33 & 0,35 \\
\hline $\mathrm{P}_{2}$ & 25,3 & 19,2 & 4,2 & 4,1 & 2,89 & 1,01 & 1,18 & 0,50 & 0,43 & 0,53 \\
\hline $\mathrm{K}_{0}$ & 25,3 & 14,1 & 4,1 & 4,3 & 2,98 & 0,78 & 1,09 & 0,40 & 0,30 & 0,30 \\
\hline $\mathrm{K}_{1}$ & 25,5 & 17,2 & 4,2 & 4,3 & 2,60 & 0,93 & 1,16 & 0,55 & 0,34 & 0,38 \\
\hline $\mathrm{K}_{2}$ & 20,4 & 15,3 & 4,3 & 4,3 & 2,69 & 0,94 & 1,31 & 0,53 & 0,40 & 0,33 \\
\hline & & & & & 200 & & & & & \\
\hline $\mathrm{N}_{0}$ & 31,6 & 14,8 & 4,9 & 4,8 & 0,49 & 0,13 & 0,12 & 0,04 & 0,52 & 0,48 \\
\hline $\mathrm{N}_{1}$ & 29,6 & 13,4 & 4,7 & 4,7 & 0,32 & 0,12 & 0,11 & 0,03 & 0,67 & 0,52 \\
\hline $\mathrm{N}_{2}$ & 28,7 & 15,1 & 4,8 & 4,7 & 0,62 & 0,14 & 0,15 & 0,04 & 0,43 & 0,62 \\
\hline $\mathrm{P}_{0}$ & 25,6 & 14,3 & 4,9 & 4,7 & 0,49 & 0,14 & 0,17 & 0,03 & 0,32 & 0,50 \\
\hline $\mathrm{P}_{1}$ & 30,5 & 14,7 & 4,8 & 4,7 & 0,39 & 0,12 & 0,12 & 0,03 & 0,63 & 0,52 \\
\hline $\mathrm{P}_{2}$ & 30,2 & 14,5 & 4,6 & 4,6 & 0,27 & 0,14 & 0,08 & 0,03 & 0,85 & 0,58 \\
\hline $\mathrm{K}_{0}$ & 34,3 & 15,3 & 4,7 & 4,6 & 0,42 & 0,11 & 0,15 & 0,03 & 0,75 & 0,52 \\
\hline $\mathrm{K}_{1}$ & 29,0 & 14,7 & 4,8 & 4,7 & 0,40 & 0,13 & 0,12 & 0,03 & 0,57 & 0,54 \\
\hline $\mathrm{K}_{2}$ & 28,9 & 13,8 & 4,9 & 4,7 & 0,28 & 0,12 & 0,09 & 0,03 & 0,73 & 0,47 \\
\hline
\end{tabular}

TABELA 5 - Teor de N total, P disponível e K trocável no Argissolo Vermelho-Amarelo da área experimental, localizada na região de Manaus-AM.

\begin{tabular}{|c|c|c|c|c|c|c|}
\hline \multirow{2}{*}{$\begin{array}{l}\text { Doses de } \\
\text { NPK }\end{array}$} & \multicolumn{2}{|c|}{ Teor de N no solo ${ }^{(1)}$} & \multicolumn{2}{|c|}{ Teor de P no solo ${ }^{(2)}$} & \multicolumn{2}{|c|}{ Teor de K no solo ${ }^{(3)}$} \\
\hline & $0-20 \mathrm{~cm}$ & $20-40 \mathrm{~cm}$ & $0-20 \mathrm{~cm}$ & $20-40 \mathrm{~cm}$ & $0-20 \mathrm{~cm}$ & $\mathrm{~cm}$ \\
\hline & \multicolumn{2}{|c|}{------g kg ${ }^{-1}$------ } & \multicolumn{2}{|c|}{------mg kg ${ }^{-1}------$} & \multicolumn{2}{|c|}{------ $\mathrm{cmol}_{\mathrm{c}} \mathrm{kg}^{-1}-------$} \\
\hline 0 & 0,83 & 0,53 & $5,00 \quad \mathrm{~b}$ & 1.84 & 0,04 & 0,03 \\
\hline 1 & 0,93 & 0,56 & $13,92 \mathrm{ab}$ & 3.91 & 0,08 & 0,06 \\
\hline 2 & 0,87 & 0,60 & $19,27 \mathrm{a}$ & 5.67 & 0,12 & 0,06 \\
\hline & \multicolumn{6}{|c|}{1999} \\
\hline 0 & 1,33 & 0,73 & $4,54 \mathrm{c}$ & $1,44 \quad b$ & 0,05 & 0,02 \\
\hline 1 & 1,46 & 0,82 & $25,35 \mathrm{~b}$ & $3,35 \mathrm{ab}$ & 0,05 & 0,03 \\
\hline 2 & 1,37 & 0,87 & 49,93 a & $5,58 \mathrm{a}$ & 0,08 & 0,06 \\
\hline \multicolumn{7}{|c|}{2000} \\
\hline 1 & 1,12 & 0,58 & $13,71 \mathrm{a}$ & 3,21 & 0,07 & 0,04 \\
\hline 2 & 1,09 & 0,62 & $21,16 \mathrm{a}$ & 7,73 & 0,05 & 0,03 \\
\hline
\end{tabular}

Médias seguidas por letras minúsculas distintas nas colunas diferem entre si, ao nível de 5\% de probabilidade, pelo teste de Tukey.

Referentes às doses de ${ }^{(1)} \mathrm{N}_{0}, \mathrm{~N}_{1}, \mathrm{~N}_{2} ;{ }^{(2)} \mathrm{P}_{0}, \mathrm{P}_{1}$ e $\mathrm{P}_{2} ;{ }^{(3)} \mathrm{K}_{0}, \mathrm{~K}_{1}$ e $\mathrm{K}_{2}$.

\section{CONCLUSÃO}

Devido ao aparecimento de fatores externos de difícil controle, durante a condução do experimento, os resultados obtidos no presente trabalho não possibilitam a recomendação oficial de adubação para o cupuaçu, mas apresentam tendências das necessidades nutricionais da cultura.

Para as duas cultivares, não há necessidade de adubação nitrogenada, assim como de adubação com doses elevadas de fósforo. Por outro lado, os menores valores de produção de frutos foram obtidos nos tratamentos com ausência de fósforo.

As respostas à aplicação de potássio foram mais lineares e positivas, principalmente para a cultivar de cupuaçu com sementes.

Nas três safras analisadas, as plantas de cupuaçu com sementes apresentaram produção em número de frutos significativamente mais elevada do que as plantas sem sementes. No entanto, em uma das três avaliadas, apesar do menor número de frutos, o peso da produção em $\mathrm{kg} /$ planta foi significativamente mais elevado nas plantas de cupuaçu sem sementes.

\section{REFERÊNCIAS BIBLIOGRÁFICAS}

ALFAIA, S.S; GUIRAUD, G.; JACQUIN, F.; MURAOKA, T.; RIBEIRO, G. A. Efficiency of ${ }^{15} \mathrm{~N}$-labelled fertilizers for rice and rye-grass cultivated in an Ultisol of Brazilian Amazonia. Biology and Fertility Soils, Berlin, v.31, n.3/4, p.329-333, 2000.

CALZAVARA, B.B.G.; MULLER, C.H.; KAHWAGE, N.C. Fruticultura tropical: o cupuaçuzeiro. Belém: EMBRAPA/CPATU, 1984. 101p

CARVALHO, J.E.U.; MULLER, C.H.; BENCHIMOL, R.L.; KATE,A.K.; ALVES, R.M. Copoasu (Theobroma grandiflorum (Willd. Ex Spreng.) Shum): Cultivo y Utilizacion. Caracas: FAO, 1999. 152p. (Manual Técnico.)

COCHRANE, T.T.; SANCHEZ, L.G.; AZEVEDO, L.G.; PORRAS, J.A.; GARVER, C.L. Land in tropical America. Brasília: CIAT/EMBRAPACPAC, 1984. v. 3. p.7-9.

CRAVO, M.S.; SMYTH, T.J. Sistemas de cultivo com altos insumos na 
Amazônia brasileira. In: SMYTH, T.J.; RAUN, W.R.; BERTSCH, F. (Ed.) Manejo de Suelos tropicais en latinoamerica. Raleigh: North Carolina state University, 1991. 144-156.

CRAVO, M.S.; SOUZA, A.G.C. Exportação de nutrientes por fruto de cupuaçuzeiro. In: REUNIÃO BRASILEIRADE FERTILIDADE DO SOLO E NUTRIÇÃO DE PLANTAS, 22., 1996, Manaus. Anais... Manaus: UFAM, 1996. p.32-633.

EMBRAPA. Manual de métodos de análise de Solo. 2. ed. Rio de Janeiro: EMBRAPA/CNPS, 1997.212p.

FASSBENDER, H.W.; ALPIZAR, L.; HEUVELDOP, J.; ENRIQUEZ, G.; FOSTER, H. Ciclos da matéria orgânica e dos nutrientes em agrossistemas com cacaueiros. In: CABALA-ROSAND, P. (Ed.). Reciclagem de nutrientes e agricultura de baixos insumos nos Trópicos. Ilhéus: CEPLAC, 1985. p.231-257.

FIGUEREDO, N.N.; MACÊDO, J.L.V.; CRAVO, M.S. Avaliação do estado nutricional do cupuaçuzeiro (Theobroma grandiflorum (Willd. Ex Spreng.) Shum) em um sistema agroflorestal na Amazônia Central. In: CONGRESSO BRASILEIRO DE SISTEMAAGROFLORESTAL, 3., 2000, Manaus, Anais... Manaus, EMBRAPA, 2000. p.48-50.

MALAVOLTA, E. ABC da adubação, São Paulo: Editora Agronômica Ceres, 1989. 292p.

MORAIS F.I.O.; SANTANA, C.J.L.; CHEPOTE, S.R.E. Resposta do cacaueiro ao nitrogênio, fósforo e potássio em solos da região cacaueira da Bahia. Revista Theobroma, Ilhéus, v.8, n.1, p.31-41, 1978.
MORAIS, F.I.O.; PEREIRA, G.C. Resposta do cacaueiro à aplicação de fertilizantes e corretivos nas condições da Amazônia. I. Crescimento e produção inicial. Revista Theobroma, Ilhéus, v.16, n.2, p.65-73, 1986.

RANZANI, G. Identificação e caracterização de alguns solos da Estação Experimental de Silvicultura Tropical do INPA. Acta Amazônica, Manaus, v.10, n.1, p.7-41, 1980.

RODRIGUES, A.C.G. O potássio na cultura do cacau. Agrotrópica, Ilhéus, v.5, n.1, p.1-12, 1983.

SANTOS, A.M.G. Aproveitamento de resíduos das culturas de cupuaçu (Theobroma grandiflorum) e pupunha (Bactris gasipae) como adubo orgânico em sistemas agroflorestais na Amazônia. 2003. 49f. Dissertação (Mestrado em Sistemas Agroflorestais) - Faculdade de Ciências Agrárias, Universidade Federal do Amazonas, Manaus, 2003.

SCHROTH, G; ELIAS, M.E.A; MACÊDO, J.L.V.; D'ANGELO, S.A.; LIBEREI, R. Growth, Yields and Mineral Nutrition of Cupuaçu (Theobroma grandiflorum) in Two Multi-strata Agroforestry Systems on a Ferralitic Amazonian Upland Soil at Four Fertilization Levels. Journal of Applied Botany, Goettingen, v.75, p.67-74, 2001.

SILVA, A.A.Q.; SILVA, H. Teores de nutrientes em cupuaçu (Theobroma grandiflorum). In: CONGRESSOBRASILEIRODE FRUTICULTURA, 8., 1986, Brasília. Anais... Brasília:SBF, 1986. p.269-271

WESSEL, M. Effects of fertilizers on growth of young cacao. Tropical Agriculture, Trinidad, v.47, n.1, p.63-66. 1970. 